\title{
Ossified Ligamentum Flavum causing Cervical Myelopathy
}

\author{
Jye Young Song, Jin Hoon Park, Sung Woo Roh \\ Department of Neurosurgery, Asan Medical Center, University of Ulsan College of Medicine, Seoul, Korea
}

Ossification of the ligamentum flavum (OLF) causing compressive cervical myelopathy or radiculopathy is rare. A 50-yearoldmale was admitted for progressive hypesthesia and paresthesia of both hands and a gradually worsening gait. MRI and CT scans demonstrated ossification of the left ligamentum flavum with dural sac and cord compression at the C5-6 level. The ossified ligamentum flavum was removed through a subtotal laminectomy and left side foraminotomy of the C5-6. Postoperatively, his gait improved remarkably and the sensory symptoms gradually resolved.

Key Words: Ligamentum flavum • Ossification • Myelopathy

\section{INTRODUCTION}

Ossification of the ligamentum flavum is a relatively wellknown spinal disorder that is most commonly observed in middle-aged men. The disease is usually seen in the thoracic or thoracolumbar regions, and is rarely observed in the cervical spine. The relative incidence of asymptomatic ossified ligamentum flavum (OLF) in the thoracic, lumbar and cervical spine is approximately $38.5,26.5 \%$, and $0.9 \%$ respectively $^{3)}$.

Although the pathogenesis is not yet fully understood, repetitive and excessive mechanical stress applied to the ligament seems to be a major factor underlying OLF development. The higher frequency of ossification in the thoracolumbar spine may be dueto the static tension placed on the ligament between the rigid rib cage above and the flexible lumbar spine below ${ }^{10)}$. Similar tension may play a role in OLF development in the cervical spine.

The present report describes a rare case of OLF at the level of C5-6 causing cervical myelopathy. This case was successfully treated using surgical methods. The report also includes a review of the relevant literature.

\section{CASE REPORT}

A 50-year-old male presented with numbness and tingling sensations in both hands that had severely aggravated in a

- Received: January 11, 2012 • Revised: March 5, 2012

- Accepted: March 12, 2012

Corresponding Author: Sung Woo Roh, MD, PhD

Department of Neurosurgery, Asan Medical Center, 388-1 Pungnap-2

dong, Songpa-gu, Seoul 138-736, Korea

Tel: +82-2-3010-3550, Fax: +82-2-476-6738

E-mail: swroh@amc.seoul.kr few dayspreviously. He reported a history of an uncomfortable gait and an inability to run due to clumsiness of the feet and weakened ankle strength for the preceding 6 months. For 3 months prior to the presentation, both hands began to feel numb and tingling, and the foot symptoms gradually worsened. He had no relevant medical or trauma history.

On admission, a general physical examination showed no abnormalities. A neurological examination identified grade IV power in flexion of both ankles and big toes, and hyperreflexia in all 4 limbs in the absence of Spurling's sign, Lhermitte's sign or Hoffman's sign. Sensory examination revealed slight diminishing in pain and temperature sensitivity below the C5 dermatome with posterior column sensation preserved.

Plain X-ray films of the cervical spine showed loss of cervical lordosis and presence of ossification of the nuchal ligament at C3-4, but no relevant findings of OLF (Fig. 1). Computerized tomography (CT) scans showed a left-sided portion of the ligamentum flavum at C5-6 was largely replaced by a high density caused by calcium (Fig. 2A and B). Magnetic resonance imaging (MRI) demonstrated that the ossification, represented by a low signal on T1- and T2-weighted images, impinged on the spinal canal resulting in nerve root and spinal cord compression at the C5-6 level. Hyperintense T2 cord signals were presented at the level of compression (Fig. 3).

A decompressive laminectomy of the C5-6 and removal of the OLF were performed using a posterior approach. The outer layer of the ligamentum flavum appeared nearly normal. Removing that normal tissue exposed an ossified lesion of 1-2 mm thickness projecting into the left neural foramina (Fig. 4). The C5-6 spinal cord and C6 nerve root were severely compressed by the OLF, which was not adhered to the dura and was relatively easy to dissect and remove without dural breech using a high speed drill and Kerrison punches.

The postoperative course was uneventful. The patient's gait 


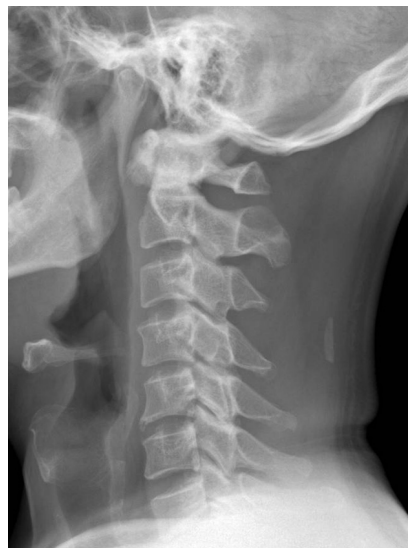

Fig. 1. Plain radiograph of the cervical spine showing loss of cervical lordotic curvature.
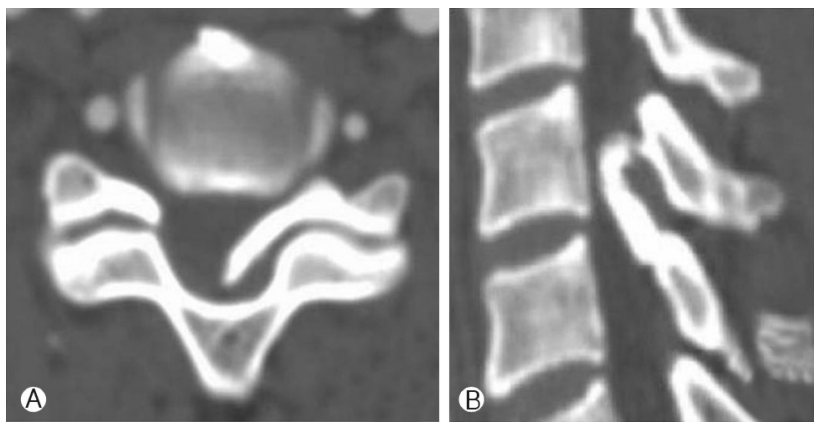

Fig. 2. Axial (A) and sagittal (B) computed tomography scans at the C5-6 level demonstrating an ossified lesion projecting into the left neural foramina.

improved greatly and the preoperative pathological sensory symptoms in both hands gradually diminished over 3 days. At 3- and 6-month follow-ups, he was able to walk and run without any discomfort, and there was no numbness or tingling in either hand.

\section{DISCUSSION}

Nuchal, cervical and thoracic posterior longitudinal ligaments and thoracolumbar flaval ligaments are the ligaments in which ossification occurs most commonly in the spine. Ossified posterior longitudinal ligaments and OLF are characterized by ectopicbone formation along the spinal ligaments and frequently lead to gradual neurological deficits or trauma-related sudden quadriplegia ${ }^{15}$. They share epidemiology, etiology and pathology in common and often coexist. However, the precise pathogenesis of OLF was not clarified until recently. According to various studies, it is now known that several systemic and local factors seem to be involved in the onset and progression of OPLL and OLF, including genetic factors, dietary habits, metabolic abnormalities andvarious re-
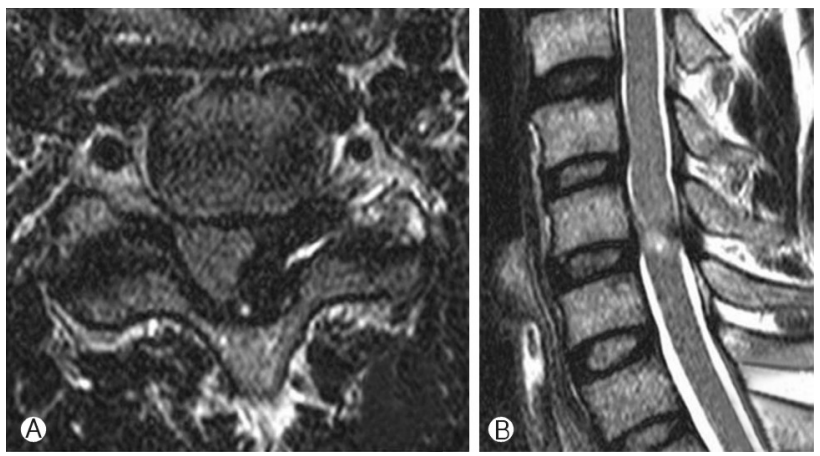

Fig. 3. T2-weighted magnetic resonance axial (A) and sagittal (B) images of the cervical spine at the C5-6 level showing OLF compressing spinal cord and left root and consequent cord signal change.

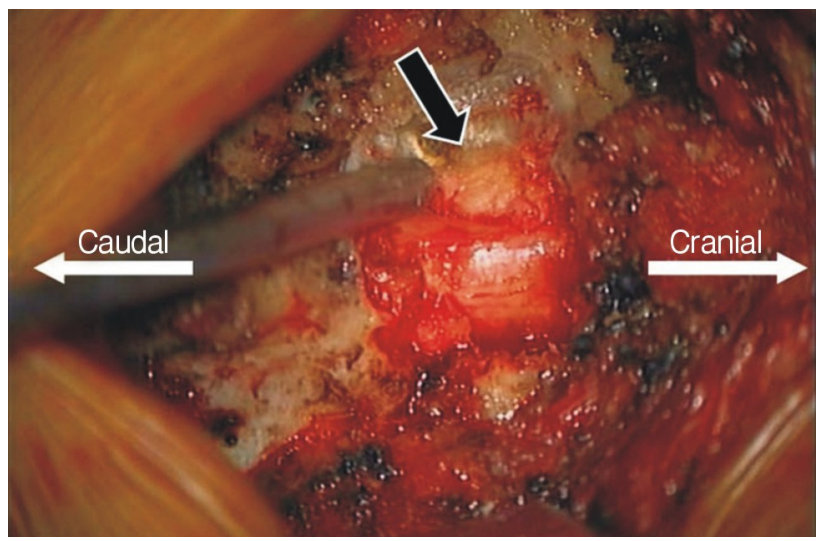

Fig. 4. Intraoperative microscopic photography of the cenvical OLF (black arrow) at the C5-6 level compressing the dural sac and spinal cord.

gional factors ${ }^{1)}$. Among them, mechanical stress is considered to play a major role in progression by inducing osteogenic differentiation in spinal ligament cells. Additionally, many studies have indicated that OPLL cells have several osteoblastic phenotypes compared with spinal ligament cells ${ }^{5,6}$.

Spinal deformity disorders, which are generally considered to put large mechanical stresses on ligaments, tend to have highrates of concurrent ossification of spinal ligaments. It has been reported that spinal balance failure and abnormal spinal curvature are highly relevant to the development of ligamentossified diseases. Miyazawa et al. ${ }^{97}$ found that patients with OLF at C2-4 had high rates of local kyphosis and an associated hyperostotic state. Otani et al. ${ }^{11)}$ also demonstrated that the incidence of OLF was higher in people with kyphosis of the thoracic or lumbar spine compared to those who did not.

It has been demonstrated that ossification can progress in patients with high segmental ROM or after surgery ${ }^{4)}$ because surgical interventions destroy the posterior structure and thus 
cause instability and even kyphosis. Li et al. ${ }^{7)}$ reported that the pathogenesis of thoracic OLF was mainly due to localized mechanical stress on the ligament. Xu et al. ${ }^{16}$ claimed that the higher prevalence of ossification in the larger stress regions indicated that there may be a stress threshold for the spinal ligaments.

OLF mainly occurs in the thoracic spine and rarely in the cervical spine. OLF was found to be the most common in the lower thoracic spine, while OPLL was usually distributed in the upper to middle thoracic region ${ }^{13)}$. The region-specific prevalence of OPLL and OLF may indicate that the segments of the ligaments under the largest mechanical stress can vary along the spine.

The terms 'ossification' and 'calcification' in regards to the ligamentum flavum have been used confusingly in the literature. Nakajima et al. reported that calcification of the cervical ligamentum flavum occurred at the C5 level predominantly in the sixth or seventh decades in Japanese females ${ }^{10}$. However, Haraguchi et al. described that unlike calcification, ossification is most commonly observed in males aged between 50 and 60, was located at the lower thoracic spine between T9 and T12, and was frequently connected to the adjacent lamina or dura. Besides, the lesionhas the "beat-like" appearance extending into the spinal neural foramen and often coexists with $\mathrm{OPLL}^{2}$. In the present case, the patient was in his fifties and had the "beat-like" lesion projecting into the left neural foramen, demonstrating the typical OLF clinical feature.

The treatment of cervical ligamentum flavum ossification is determined on the same basis as that of other spinal diseases. While conservative treatment of orthosis and use of analgesics is recommended for patients without neurological deficiency or disease progression, surgery may be required for patients with persistent or aggravating neurological symptoms ${ }^{14)}$.

The surgical treatment for OLF is a bilateral decompressive laminectomy and removal of the OLF. Additionally, internal fixation is performed in some centers. In 2 similar studies, therates of development of progressive deformity after laminectomy were reported to range from $14-47 \%{ }^{12)}$ and $21-42 \%{ }^{8}$, respectively. Yang et al. claimed that cervical laminectomy may pose a risk of changing the focal mechanics and therefore lead to ossification of other ligaments at the same level or ligaments at adjacent levels, even if postoperative deformity does not occur ${ }^{17}$. However, the indication for instrumentation remains controversial. The preservation of the paraspinal muscles, fascias and cervical spine motion is important for reducing postoperative axial neck pain and for maintaining cervical motion, as well as for preventing postoperative kyphotic change ${ }^{8}$. Therefore, laminectomy with posterior fixation should be used judiciously based on a surgeon's experience.

In the current case, a posterior-approach decompressive laminectomy at the C5-6 and removal of the OLF was performed without posterior fixation. By the 6th postoperative month, the patient showed complete recovery and no signs of recurrence. Radiological images at later postoperative times are required before complete success can be considered achieved.

\section{CONCLUSION}

Reports of cervical OLF are rare, and its pathogenesis is not clearly understood. We experienced a case of cervical OLF causing cervical myelopathy, and reviewed the literature regarding possible causes and treatments. The patient underwent a decompressive cervical laminectomy and removal of the OLF, and this resulted in a good postoperative outcome.

\section{REFERENCES}

1. Cho KH: Surgery of Ossification of Posterior Longitudinal Liga ment (OPLL) of Cervical Spine. Korean J Spine 1:1-15, 2004

2. Haraguchi K, Yamaki T, Kurokawa Y, Ohtaki M, Ibayashi Y, Uede T, et al: A case of calcification of the cervical ligamentum flavum]. No Shinkei Geka 24:69-73, 1996

3. Hasue M, Kikuchi S, Fujiwara M, Sakuyama Y, Sakamoto T, Miura H, et al: Roentgenographic analysis of ossification ofthe spinal ligament with special reference to the finding of the whole spine. Seikei Geka 31:1179-1186, 1980

4. Hirabayashi K, Watanabe K, Wakano K, Suzuki N, Satomi K, Ishii Y: Expansive open-door laminoplasty for cervical spinal stenotic myelopathy. Spine (Phila Pa 1976) 8:693-699, 1983

5. Inaba K, Matsunaga S, Ishidou Y, Imamura T, Yoshida H: Effect of transforming growth factor-beta on fibroblasts in ossification of the posterior longitudinal ligament. In Vivo 10:445449, 1996

6. Kon T, Yamazaki M, Tagawa M, Goto S, Terakado A, Moriya $\mathrm{H}$, et al: Bone morphogenetic protein-2 stimulates differentiation of cultured spinal ligament cells from patients with ossification of the posterior longitudinal ligament. Calcif Tissue Int 60:291-296, 1997

7. Li F, Chen Q, Xu K: Surgical treatment of 40 patients with thoracic ossification of the ligamentum flavum. J Neurosurg Spine 4:191-197, 2006

8. Lu JJ: Cervical laminectomy: technique. Neurosurgery 60: S149-153, 2007

9. Miyazawa N, Akiyama I: Ossification of the ligamentum flavum of the cervical spine. J Neurosurg Sci 51:139-144, 2007

10. Nakajima K, Miyaoka M, Sumie H, Nakazato T, Ishii S: Cervical radiculomyelopathy due to calcification of the ligamenta flava. Surg Neurol 21:479-488, 1984

11. Otani K, Aihara T, Tanaka A, Shibasaki K: Ossification of the ligamentum flavum of the thoracic spine in adult kyphosis. Int Orthop 10:135-139, 1986

12. Ryken TC, Heary RF, Matz PG, Anderson PA, Groff MW, 
Holly LT, et al: Cervical laminectomy for the treatment of cervical degenerative myelopathy. J Neurosurg Spine 11:142149, 2009

13. Sato T, Kokubun S, Tanaka Y, Ishii Y: Thoracic myelopathy in the Japanese: epidemiological and clinical observations on the cases in Miyagi Prefecture. Tohoku J Exp Med 184:1-11, 1998

14. Sonntag VK: Ossification of the ligamentum flavum (OLF): an increasing cause of cervical myelopathy. World Neurosurg 75:445-446, 2011

15. Sugimoto $\mathrm{Y}$, Ito $\mathrm{Y}$, Tanaka M, Tomioka M, Hasegawa $\mathrm{Y}$,
Nakago K, et al: Cervical cord injury in patients with ankylosed spines: progressive paraplegia in two patients after posterior fusion without decompression. Spine (Phila Pa 1976)34: E861-863, 2009

16. Xu P, Wang C, He H, Xu G, Ye X: Spinal balance failure: a potential cause of spinal ligament ossification. Med Hypotheses 76:908-910, 2011

17. Yang J, Ni B, Xie N, Guo Q, Wang L: Surgical treatments of myelopathy caused by cervical ligamentum flavum ossification. World Neurosurg 75:546-550, 2011 